\title{
Genome-Wide Analysis of Fatty Acid Desaturases in Soybean (Glycine max)
}

\author{
Xiaoyuan Chi • Qingli Yang • Yandu Lu • \\ Jinyan Wang • Qingfen Zhang • Lijuan Pan • \\ Mingna Chen • Yanan He • Shanlin Yu
}

Published online: 11 January 2011

(C) Springer-Verlag 2011

\begin{abstract}
Fatty acid desaturases can introduce double bonds into the hydrocarbon chains of fatty acids to produce unsaturated fatty acids. In the present study, 29 full-length desaturase genes were identified from soybean genome by a thorough annotation exercise. A comprehensive analysis was performed to characterize phylogeny, chromosomal locations, structures, conserved motifs, and expression patterns of those genes. The soybean genes were phylogenetically clustered into nine subfamilies with the Arabidopsis counterparts, FAB2, FAD2, FAD3, FAD5, FAD6, FAD7, FAD8, SLD1, and DES1. Twenty-nine desaturase genes were found to be distributed on at least 15 of the 20 soybean chromosomes. The gene structures and motif compositions were considerably conserved among the subfamilies. The majority of desaturase genes showed specific temporal and spatial expression patterns across
\end{abstract}

Xiaoyuan Chi and Qingli Yang contributed equally to the work.

X. Chi $\cdot$ Q. Yang $\cdot$ L. Pan $\cdot$ M. Chen $\cdot$ Y. He $\cdot S$. Yu $(\bowtie)$

Shandong Peanut Research Institute,

Qingdao 266100, People's Republic of China

e-mail: yushanlin1956@yahoo.com.cn

Y. $\mathrm{Lu}$

Qingdao Institute of Bioenergy and Bioprocess Technology,

Chinese Academy of Sciences,

Qingdao 266101, People's Republic of China

J. Wang

State Key Laboratory of Crop Genetics and Germplasm

Enhancement, College of Horticulture,

Nanjing Agricultural University,

Nanjing 210095, People's Republic of China

Q. Zhang

Sino-Danish Renewable Energy Development Programme,

Beijing 100038, People's Republic of China different tissues and developmental stages based on microarray data analyses. The study may provide new insights into the origin and evolution of fatty acid biosynthesis pathways in higher plants. Additionally, the characterization of desaturases from soybean will lead to the identification of additional genes for genetic modification of plants to produce nutritionally important fatty acids.

Keywords Glycine max - Genome · Fatty acid desaturase . Phylogenetic analysis
Abbreviations
FAB2 Stearoyl-ACP desaturase
FAD2 Microsomal $\Delta 12$ desaturase
FAD3 Microsomal $\omega 3$ desaturase
FAD5 $\Delta 7$ Desaturase
FAD6 Plastidial $\Delta 12$ desaturase
FAD7 Plastidial $\omega 3$ desaturase
FAD8 Plastidial $\omega 3$ desaturase
ADS $\quad \Delta 9$ Desaturase
SLD1 Sphingolipid $\Delta 8$ desaturase
DES1 Sphingolipid $\Delta 4$ desaturase
D6D $\Delta 6$ Desaturase
D5D $\Delta 5$ Desaturase
DesC Cyanobacterial $\Delta 9$ desaturase
DesA Cyanobacterial $\Delta 12$ desaturase
DesB Cyanobacterial w3 desaturase

\section{Introduction}

Fatty acids, a major source of energy in plant, are the main compositions of plant membrane lipids and seed storage 
lipids (Topfer et al. 1995; Ohlrogge and Browse 1995). The oxidative stability and nutritional value of seed oils, which are already being considerable of commercial importance, are also affected by the levels of fatty acids (Pattee et al. 2002)

The biosynthesis of polyunsaturated fatty acids (PUFAs) in different organisms involves a variety of pathways, which are catalyzed by a complex series of desaturation and elongation steps ( $\mathrm{Li}$ et al. 2010). Fatty acid desaturases are enzymes that introduce double bonds into the hydrocarbon chains of fatty acids (Singh et al. 2002). They contain three conserved histidine regions with the exception of stearoylacyl carrier protein (ACP) gene, which contains just two histidine regions (Tao et al. 2006; Shilman et al. 2010). Histidine-rich boxes are thought to form a part of the diiron center where oxygen activation and substrate oxidation occur (Chi et al. 2008a, b; Lu et al. 2009). The desaturation processes take place in both the plastidial membrane and the endoplasmic reticulum (ER) membrane through two different pathways (Ohlrogge and Browse 1995). The genes for ER- and plastid-derived desaturases have been characterized from many plant species to date, capable of introducing double bonds at various locations on the fatty acyl chain (Chi et al. 2008a, b; Lu et al. 2010; Chen et al. 2010)

Soybean (Glycine max) cultivated for both high seed protein $(42 \%)$ and oil contents $(23 \%)$ since the beginning of humanity has long been one of the most important economical crops all over the world (Dornbos and Mullen 1992; Saski et al. 2005; Zhang et al. 2008a; Vijay et al. 2009). The functional and nutritional value of soybean oil is determined to a large extent by the degree of saturation of triacylglycerol fatty acids. With better understanding of fatty acid metabolic pathways, many desaturase genes with different regioselectivities have been cloned from soybean. A plastidial $\omega 6$ desaturase, designated as GmFAD6 (Glyma02g36460), has been cloned from soybean (William et al. 1994). Three different soybean omega-6 desaturase genes, GmFAD2-1, GmFAD2-2, and GmFAD2-3, have been reported (Heppard et al. 1996; Tang et al. 2005; Li et al. 2007). GmFAD2-1A (Glyma10g42470) and GmFAD2-1B (Glyma20g24530) genes, specifically expressed in developing seeds, played a predominant role in determining the PUFA content of seed storage oil. In contrast, GmFAD2-2 and GmFAD2-3 were constitutively expressed in both vegetative tissue and developing seeds. Recent soybean genomic analyses have shown that GmFAD2-2 gene exists as three alleles, GmFAD2-2A (Glyma19g32930), GmFAD2-2B (Glyma19g32940), and GmFAD2-2C (Glyma03g30070; Schlueter et al. 2007). Transcript abundance of GmFAD2$2 C$ was observed to increase approximately eightfold in pods grown in cool conditions relative to those in warmer conditions. The third gene, GmFAD2-3, was also constitutively expressed in both vegetative and developing seed tissues but showed no significant changes in transcript levels in cold-stressed leaves ( $\mathrm{Li}$ et al. 2007). Bilyeu et al. (2003) identified three independent microsomal omega-3 fatty acid desaturases in soybean, GmFAD $3 A$ (Glyma14g37350), GmFAD3B (Glyma02g39230), and GmFAD3C (Glyma18g06950). GmFAD3A was significantly upregulated in developing seeds whereas GmFAD $3 B$ and $G m F A D 3 C$ levels remained relatively low in that tissue. A significant downregulation of $\alpha$-linolenic acid was achieved by siRNA-mediated silencing of three active members of the FAD3 family in soybean (Flores et al. 2008). Furthermore, the silencing was stably inherited in engineered soybean lines. Three alleles of GmFAB2 have been identified and characterized from soybean. Transcripts of the GmFAB2A (Glyma07g32850) and GmFAB2B (Glyma02g15600) were detected most abundantly in developing seeds; however, differences in transcript abundance between A and B were not dramatic (Byfield et al. 2006). High levels of GmFAB2C (Glyma14g27990) transcripts were expressed only in developing seeds, and mutations in this gene from two soybean germplasm sources, mutants A6 (30\% stearate) and FAM94-41 (9\% stearate), resulted in elevated seed stearic acid (Zhang et al. 2008b). Two FAD7 paralogous genes have been identified in soybean, GmFAD7-1 (Glyma18g43210) and GmFAD7-2 (Glyma07g18350; Andreu et al. 2010). Both GmFAD7-1 and GmFAD7-2 genes were expressed in all soybean tissues examined, displaying their highest mRNA accumulation in leaves. This expression profile contrasted with $G m F A D 3 A$ and $G m F A D 3 B$ mRNA accumulation, which was very low in this tissue.

Currently, the soybean genome has been sequenced, approximately $975 \mathrm{Mb}$ captured in 20 chromosomes, and its gene prediction and annotation are publicly available. Soybean is a well-documented paleopolyploid for which there is molecular evidence of genome duplications that occurred at approximately 59 and 13 Ma ago (Schmutz et al. 2010). The availability of the genome and annotated genes facilitates comparative genomic studies which will help to address major plant biology questions in silico and provide an effective means of gene discovery in focused metabolic situations (Bowman et al. 2007). In the present study, orthologous genes encoding enzymes of fatty acid desaturation from soybean were identified. The identification of novel desaturases will be helpful for the reconstruction of the pathways involved in unsaturated fatty acid biosynthesis and metabolic engineering of fatty acid synthesis in soybean. Moreover, the characterization of desaturases from soybean will provide ample candidate genes for the production of nutritionally important fatty acids in transgenic plants. 


\section{Materials and Methods}

Gene Retrieval and Annotation

The genome of G. max was downloaded from the Phytozome database (http://www.phytozome.net/soybean), while the Arabidopsis data were available from The Arabidopsis Information Resource (TAIR version 9.0). An initial set of query proteins, including well-characterized and putative fatty acid desaturases identified from Arabidopsis, soybean, and other plants, were obtained from GenBank database (National Center for Biotechnology Information). Each protein in this query dataset was used to search the potential novel sequences in soybean and Arabidopsis for which whole genome sequences were available by BLASTP and TBLASTN programs, with an $E$-value $<1 \mathrm{e}^{-10}$. The searches were iterated until convergence. As for the incorrectly predicted genes, manual reannotation was performed using online web server FGENESH (http://inux1.softberry.com/berry.phtml; Salamov and Solovyev 2000). The putative desaturase genes across soybean genome were listed in Table 1. The other amino acid sequences beyond soybean were retrieved from GenBank and summarized in Table 2. The Arabidopsis and soybean proteome data included alternatively splicing variants. We counted these splicing variants of the two organisms as one gene in our statistics in Tables 1 and 2. For instance, Glyma20g24530 had five known splicing variants (Glyma20g24530.1, Glyma20g24530.2, Glyma20g24530.3, Glyma20g24530.4, and Glyma20g24530.5); only one protein was included in our phylogenetic analyses; we counted them as one single gene in Table 1.

\section{Chromosomes Mapping and Gene Structure Prediction}

Fatty acid desaturase genes were mapped on chromosomes by determining their chromosomal positions given in the Phytozome soybean database. Gene structure display server program (Guo et al. 2007) was used to illustrate exon/intron organization for individual desaturase gene by comparison of the cDNAs with their corresponding genomic DNA sequences from Phytozome (http://www.phytozome.net/soybean).

\section{Conserved Motif Scanning}

To identify the conserved motifs, Multiple Expectation Maximization for Motif Elicitation (MEME) version 2.2 (Bailey and Elkan 1995) was employed with a set of parameters as follows: number of repetitions - any, maximum number of motifs - 20, and optimum motif width set to $\geq 6$ and $\leq 200$. The motifs obtained were annotated using SMART (http://smart.embl-heidelberg.de/) and National Center for Biotechnology Information Conserved Domain Database search program.
SSR Loci Screening

The SSR loci were screened in the 41 fatty acid desaturase genes by MISA software (MicroSAtellite, http://pgrc.ipk-gatersleben.de/misa/). The di-, tri-, tetra-, and penta-nucleotide SSR motifs were used in the sequence analysis. SSR motifs, which repeated more than seven times for di-nucleotide, five for tri-nucleotide, four for tetra-nucleotide, and penta-nucleotide, were identified (Thiel et al. 2003).

\section{Phylogenetic Analysis}

Sequence alignments were generated using the ClustalW program (Thompson et al. 1994). The produced files with "*.aln" extension were opened by BioEdit at the option of "Graphic View." Conserved histidine-rich boxes were identified manually from the BioEdit sequence editor. Phylogenetic analyses were conducted using MEGA version 4 (Tamura et al. 2007). The neighbor-joining phylogenetic tree was constructed with default parameters using complete protein sequences of desaturase genes. Bootstrap with 1,000 replicates was used to establish the confidence limit of the tree branches. Three different protein targeting prediction programs were used to estimate the putative subcellular locations of the candidate proteins: IPSORT (http://hc.ims.u-tokyo.ac.jp/iPSORT/), TARGETP (http:// www.cbs.dtu.dk/services/TargetP/), and PREDOTAR (http://urgi.versailles.inra.fr/predotar/predotar.html).

\section{Coexpression Analysis}

The gene coexpression patterns were examined using tools at the Genevestigator web site (Zimmermann et al. 2005; https://www.genevestigator.ethz.ch/). For genes with more than one probe set, the highest of expression values was considered. When several genes had the same probe set, then they were considered to have same level of transcript abundance. The expression data were gene-wise normalized and hierarchical clustered based on Pearson coefficients with average linkage in the Genesis (version 1.75) program (Sturn et al. 2002).

\section{Results}

Identification and Organization of Fatty Acid Desaturase Genes

Forty-one desaturase genes were predicted and annotated from the soybean genome using BLASTP and TBLASN programs with the query sequence. The candidate genes identified in this study were listed in Table 1 . Twelve partial 


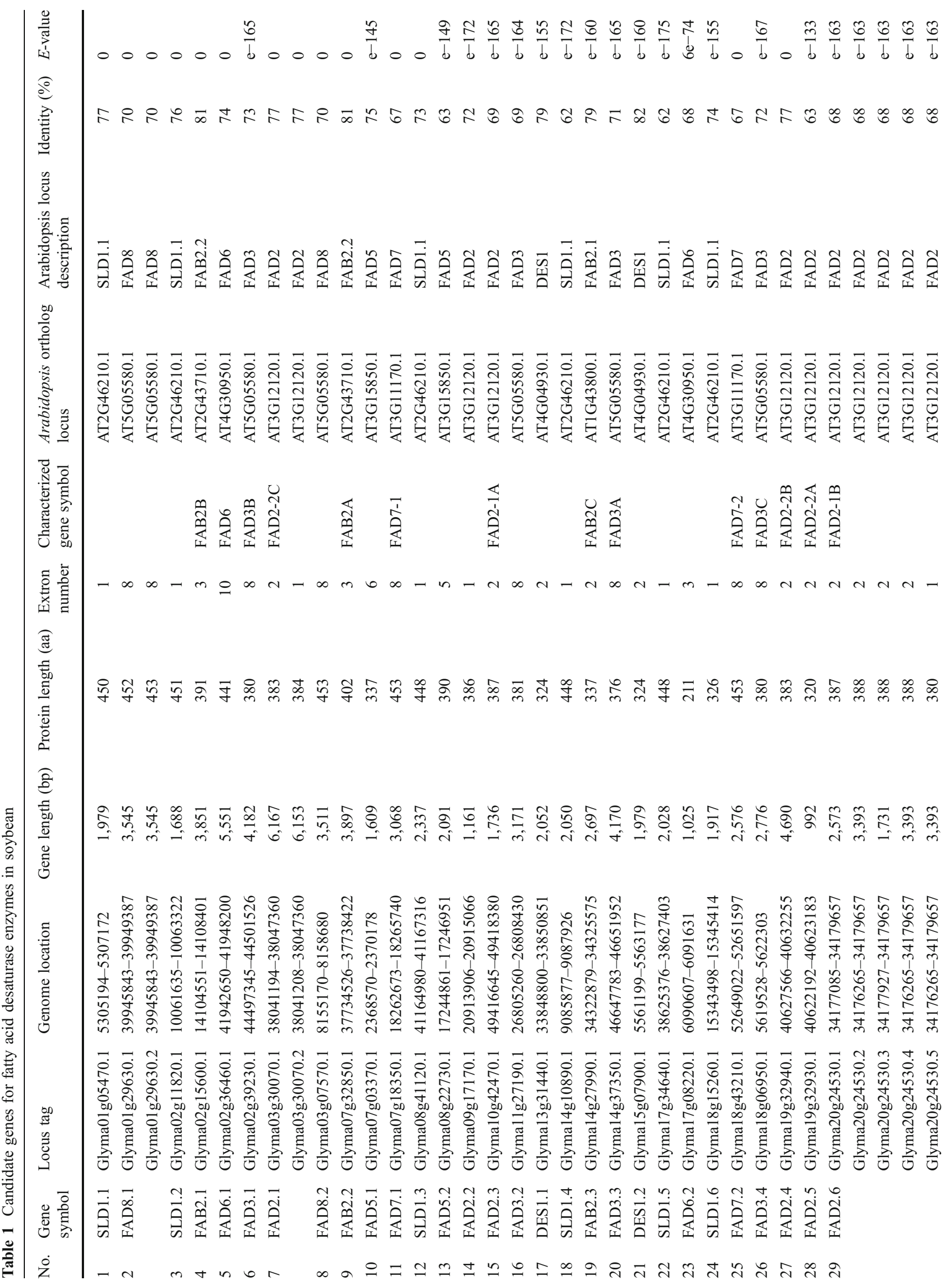


অ

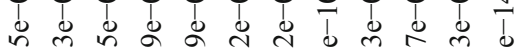

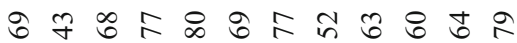
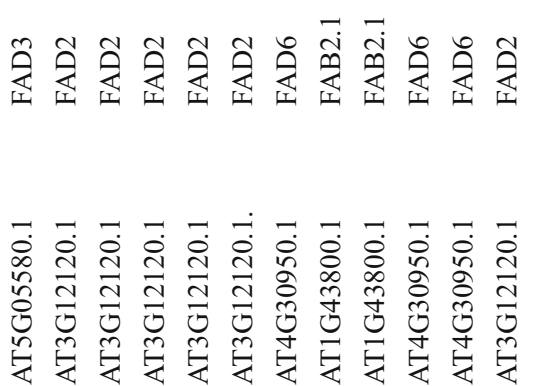

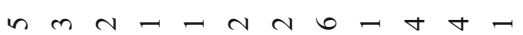

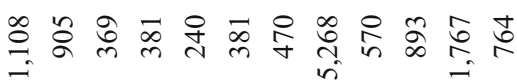

총ำำ

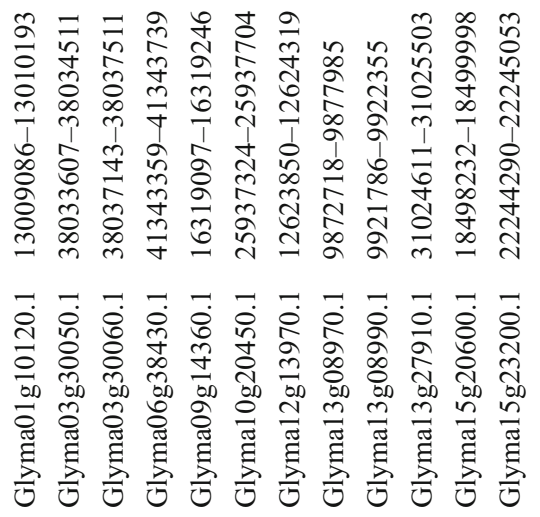

ஜิ sequences existed in the obtained soybean putative desaturase genes, including six FAD2, three FAD6, two FAB2, and one FAD3 gene. To facilitate the classification and phylogenetic analyses of different types of desaturases, these partial sequences were excluded in the following study. The remaining 29 full-length genes contained typical histidine-rich boxes (Table 3), which accorded with the standard of different types of desaturase genes. For example, all the genes from FAD6 subfamily matched the standard for plastidial $\Delta 12$ desaturase, i.e., GHDCXH, $\mathrm{HX}_{2} \mathrm{HH}$, and $\mathrm{HXPHH}$; two histidine-boxes of FAB2 genes were consistent with these of plastidial stearoyl-ACP desaturases that were represented as EENRHG, DEKRHE. Compared to Arabidopsis, soybean appeared to possess multiple copies of the gene due to the consequence of repeated rounds of genome duplication followed by limited sequence loss (Schmutz et al. 2010).

Figure 1 showed the distribution of the fatty acid desaturase genes on different soybean chromosomes. As for Arabidopsis thaliana, 18 fatty acid desaturase genes were found to distribute on five chromosomes. Seven genes were located on chromosome 3 , four on chromosome 2, three on chromosome 5, and two on chromosomes 1 and 4. As shown in Fig. 1, 29 soybean desaturase genes were found to be distributed on at least 15 of the 20 chromosomes. Substantial clustering of these genes was evident on several chromosomes. The maximum number of genes was located on chromosome 2 as four. Three genes were located on chromosomes 7, 14, and 18; two on chromosomes $1,3,8,17$, and 19; and one on chromosomes $9,10,11,13,15$, and 20 .

Different members of many fatty acid gene subfamilies were located in different soybean chromosomes. This was observed for the FAB2, FAD2, FAD3, FAD7, FAD8, DES1, and SLD1 gene subfamilies. For instance, different gene members of $F A B 2$ subfamily were located on chromosomes 2,7 , and 14, respectively. For the SLD1 subfamily, different members were located on chromosomes 1, 2, 8, 14, 17, and 18 , respectively. Almost all the members of each subfamily were distributed in different chromosomal regions except that GmFAD2.4 and GmFAD2.5 were tandemly duplicated within the same small chromosomal region. This indicated that fatty acid desaturase genes were distributed widely in the soybean genome and they might originate from different ancestors.

\section{Gene Structures and Distribution of Conserved Motifs}

The MEME motif search tool was employed to identify the conserved motifs presenting in soybean and Arabidopsis fatty acid desaturases (Fig. 2), and 20 distinct motifs were identified. Most of the motifs belonged to the coiled coil region (transmembrane) and a region of unknown com- 
Table 2 List of organisms and desaturase protein sequences analyzed in this study (except for the above sequences from soybean genome)

\begin{tabular}{|c|c|c|}
\hline Organisms & Accession & $\begin{array}{l}\text { Gene } \\
\text { symbol }\end{array}$ \\
\hline \multirow[t]{21}{*}{ Arabidopsis thaliana } & AT1G43800.1 & FAB2.1 \\
\hline & AT2G43710.1 & FAB 2.2 \\
\hline & AT2G43710.2 & FAB 2.2 \\
\hline & AT3G02610.1 & FAB 2.3 \\
\hline & AT3G02620.1 & FAB 2.4 \\
\hline & AT3G02630.1 & FAB2.5 \\
\hline & AT5G16230.1 & FAB2.6 \\
\hline & AT5G16240.1 & FAB2.7 \\
\hline & AT1G06080.1 & ADS.1 \\
\hline & AT2G31360.1 & ADS.2 \\
\hline & AT3G12120.1 & FAD2 \\
\hline & AT3G12120.2 & FAD2 \\
\hline & AT4G30950.1 & FAD6 \\
\hline & AT3G11170.1 & FAD7 \\
\hline & AT5G05580.1 & FAD8 \\
\hline & AT2G29980.1 & FAD3 \\
\hline & AT2G29980.2 & FAD3 \\
\hline & AT3G15850.1 & FAD5 \\
\hline & AT2G46210.1 & SLD1.1 \\
\hline & AT3G61580.1 & SLD1.2 \\
\hline & AT4G04930.1 & DES1 \\
\hline \multirow[t]{5}{*}{ Chlamydomonas reinhardtii } & EDP04705 & FAB2 \\
\hline & EDP04777 & FAD2 \\
\hline & EDP03637 & FAD6 \\
\hline & EDP09401 & FAD7 \\
\hline & EDP02613 & FAD5 \\
\hline \multirow[t]{3}{*}{ Homo sapiens } & BAG35237 & ADS \\
\hline & AAD20018 & D6D \\
\hline & AAF29378 & D5D \\
\hline \multirow[t]{3}{*}{ Rattus norvegicus } & EDL94279 & ADS \\
\hline & BAA75496 & D6D \\
\hline & AAG35068 & D5D \\
\hline \multirow[t]{3}{*}{ Synechocystis sp. PCC 6803} & BAA03982 & DesC \\
\hline & BAA18169 & DesA \\
\hline & BAA02924 & DesB \\
\hline \multirow[t]{3}{*}{ Brassica napus } & AAF78778 & FAD2 \\
\hline & AAA50157 & FAD6 \\
\hline & CAA11857 & SLD1 \\
\hline \multirow[t]{2}{*}{ Borago officinalis } & AAG43277 & SLD1 \\
\hline & AAD01410 & D6D \\
\hline \multirow[t]{2}{*}{ Synechococcus sp. PCC 7002} & AAF21445 & DesA \\
\hline & AAB61352 & DesB \\
\hline \multirow[t]{2}{*}{ Chlorella vulgaris } & BAB78716 & FAD2 \\
\hline & BAB78717 & FAD3 \\
\hline \multirow[t]{2}{*}{ Ostreococcus lucimarinus } & ABO95591 & FAB2 \\
\hline & ABP00213 & FAD5 \\
\hline Mortierella alpina & CAB38177 & ADS \\
\hline
\end{tabular}

Table 2 (continued)

\begin{tabular}{lll}
\hline Organisms & Accession & $\begin{array}{l}\text { Gene } \\
\text { symbol }\end{array}$ \\
\hline Acheta domesticus & AAK25797 & ADS \\
Synechococcus elongatus PCC 7942 & ABB58591 & DesC \\
Haematococcus pluvialis & ABP57425 & FAB2 \\
Arachis hypogaea & ACI45450 & FAB2 \\
Lotus corniculatus & AAY78547 & FAB2 \\
Linum usitatissimum & CAA07349 & FAB2 \\
Bassia scoparia & AAL26877 & FAB2 \\
Cyprinus carpio & CAB57858 & ADS \\
Caenorhabditis elegans & AAF97550 & ADS \\
Drosophila simulans & CAB52475 & ADS \\
Gallus gallus & CAA42997 & ADS \\
Saccharomyces cerevisiae & AAA34826 & ADS \\
Cyanidioschyzon merolae & BAA28834 & ADS \\
Phaeodactylum tricornutum & AAW70158 & ADS \\
Oryza sativa & EEE57453 & DES1 \\
Limnanthes douglasii & AAG28599 & D5D \\
Danio rario & Q9DEX7 & D5D/D6D \\
Echium pitardii & AAL23581 & D6D \\
Thalassiosira pseudonana & AY817154 & SLD1 \\
Helianthus annuus & CAA60621 & SLD1 \\
\hline
\end{tabular}

plexity which represented the typical domains of desaturase as indicated by SMART. No common motifs were found in all the members of the fatty acid desaturase family. All proteins belonging to FAD2 subfamily had the motifs 2, 5, $6,15,16$, and 17, except for GmFAD2.5, which did not have motif 17. All FAD3/FAD7/FAD8 subfamily had the motifs 2, 3, 5, and 14, except for GmFAD7.2, which had another motif 13 . All FAB2 subfamily members possessed the motifs $1,8,9,13$, and 20 , whereas the motif 9 was absent in GmFAB2.3. All SLD1 proteins had the motifs 4, 6, $10,12,14$, and 18, except for GmSLD1.6, which was lack of motifs 10 and 18 . The conserved motifs 7 and 11 were presented only in the FAD5/ADS and DES1 subfamily members, respectively.

Genes in the same subfamily had more similar exon/ intron structures than those genes in the other subfamilies (Fig. 2). The FAD3/FAD7/FAD 8 subfamily members mostly had up to eight exons whereas the FAB2 subfamily members possessed two to four exons. Members of DES1 subfamily had two exons, compared to the FAD5/ADS subfamilies, which possessed five to six exons. All of FAD2 and SLD1 subfamily members had one exon, except for GmFAD2.5, which possessed two exons. The FAD6 subfamily members usually possessed ten exons, whereas three exons were present in GmFAD6.2. 
Table 3 Conserved histidine-rich boxes of fatty acid desaturases in soybean

\begin{tabular}{llll}
\hline Name & H-box1 & H-box2 & H-box3 \\
\hline FAB2 & WTRAWTAEENRHGDL & AA(S)DEKRHE & $/$ \\
FAD2 & GVWVIAHECGHHAFS & VPYFSWKYSHRRHHSNTGSL & ITD(G)THVA(V)HHLFSTMPH \\
FAD6 & FVIGHDCAHRSFS & EPWRFKHDRHHAKTN & HDINVHIPHHISP \\
FAD3/FAD7/FAD8 & TMFWALFVLGHDCGHGSFS & YHGWRISHRTHHQN(H)HGH & HHDIGTHVIHHLFPQIPHYHL \\
FAD5 & GITLSFHRNLSHRSFK & QGNPIDWVSTHRYHHQFCD & GEGWHNNHHAFEYSAR \\
SLD1 & GHDSGHY & WWKWTHNAHHIA(S)CNSLD & GGLQFQLEHHLFPRLPR \\
DES1 & NLFLAIHELSHNLAF & TFQKYHLEHHRFQGVDGID & VGYHNEHHDFPRIPG \\
\hline
\end{tabular}

SSR Markers Development and Marker-Assisted Breeding

During the last decade, microsatellites or SSRs have been proven to be useful markers in plant genetic research and have been used for marker-assisted breeding purposes (Varshney et al. 2005). SSR markers have some advantages, such as (1) highly informative, (2) locus-specific and frequently show co-dominant inheritance, (3) adaptable to high-throughput genotyping, and (4) simple to maintain and distribute (Yu et al. 2004). Unfortunately, the development of SSR markers is expensive, labor-intensive, and timeconsuming if SSRs are developed from genomic libraries (Senthilvel et al. 2008). With the completion of soybean genomic sequences, the development of SSR markers will be facilitated in a large-scale level.

By MISA analysis, there were eight SSR loci derived from seven different genes out of 41 candidate genes involved in fatty acid biosynthesis (Table 4). All these SSRs could be divided into four kinds of motifs, such as di- nucleotide, tri-nucleotide, tetra-nucleotide, and pentanucleotide. The di- and tri-nucleotide motifs were the most frequent motifs in eight SSR loci. These SSR loci were distributed in the $5^{\prime}$ UTR, intron, and extron. Five SSR loci were located in the $5^{\prime}$ UTR, two in the intron, and one in the extron. These SSR markers could help geneticists and breeders identify fatty acid biosynthesis genes in different accessions and cultivars in order to screen the available soybean crops which hold nutritional value of seed oils.

Phylogenetic Analysis of Fatty Acid Desaturase Genes from Soybean Genome

The polyunsaturated fatty acids are synthesized by two distinct pathways in plants, known as the prokaryotic and eukaryotic pathways, which are located within the plastid and the endoplasmic reticulum, respectively (Sato and Moriyama 2007). Therefore, two major classes of desaturases exist: soluble and membrane-bound desaturases.

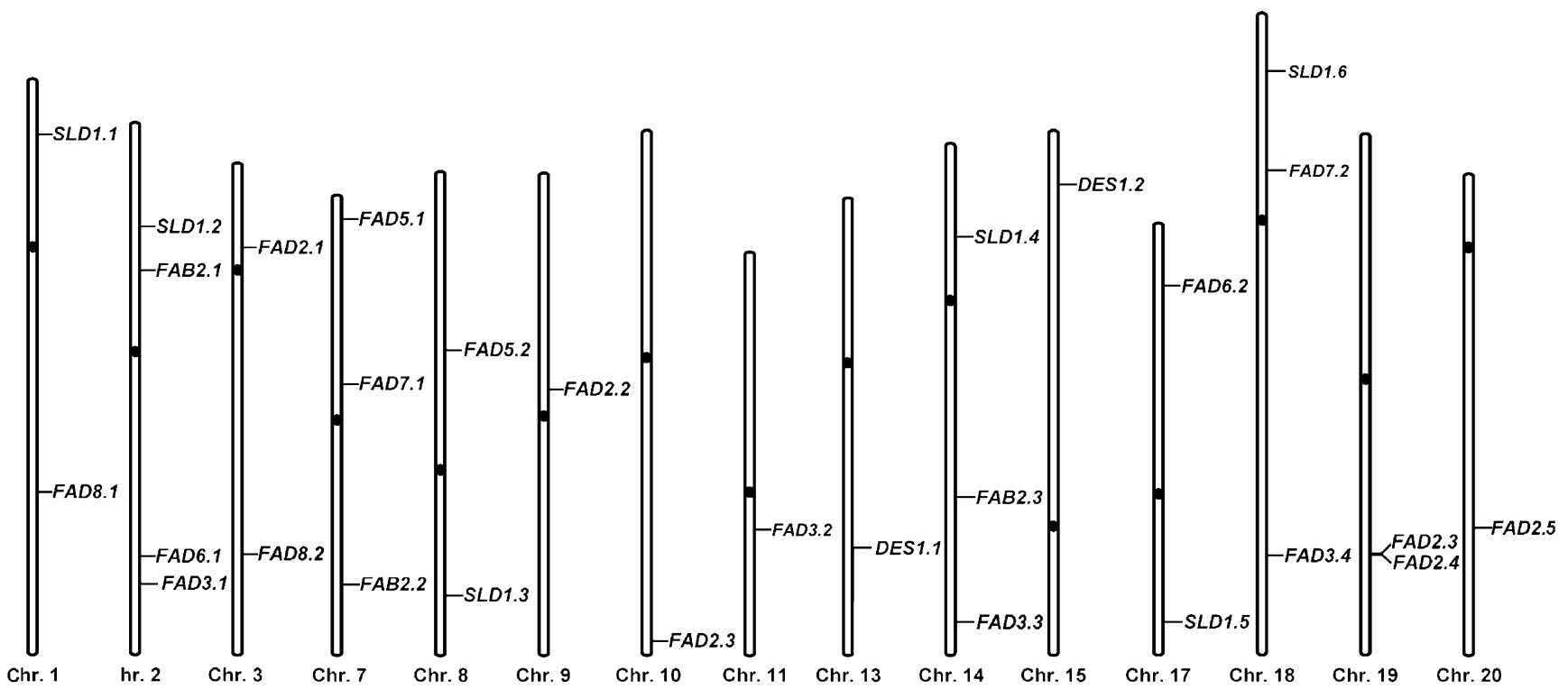

Fig. 1 Chromosomal locations of soybean fatty acid desaturase genes. Only those chromosomes bearing fatty acid desaturase genes (15) were represented. Centromeric region was drawn in black. Genes were plotted according to their sequence positions along the chromosomes.
Chromosome numbers corresponded to soybean linkage groups as follows: 1 D1a, $2 \mathrm{D} 1 \mathrm{~b}, 3 \mathrm{~N}, 7 \mathrm{M}, 8 \mathrm{~A} 2,9 \mathrm{~K}, 10 \mathrm{O}, 11 \mathrm{~B} 1,13 \mathrm{~F}, 14$ B2, $15 \mathrm{E}, 17 \mathrm{D} 2,18 \mathrm{G}, 19 \mathrm{~L}, 20 \mathrm{I}$ 

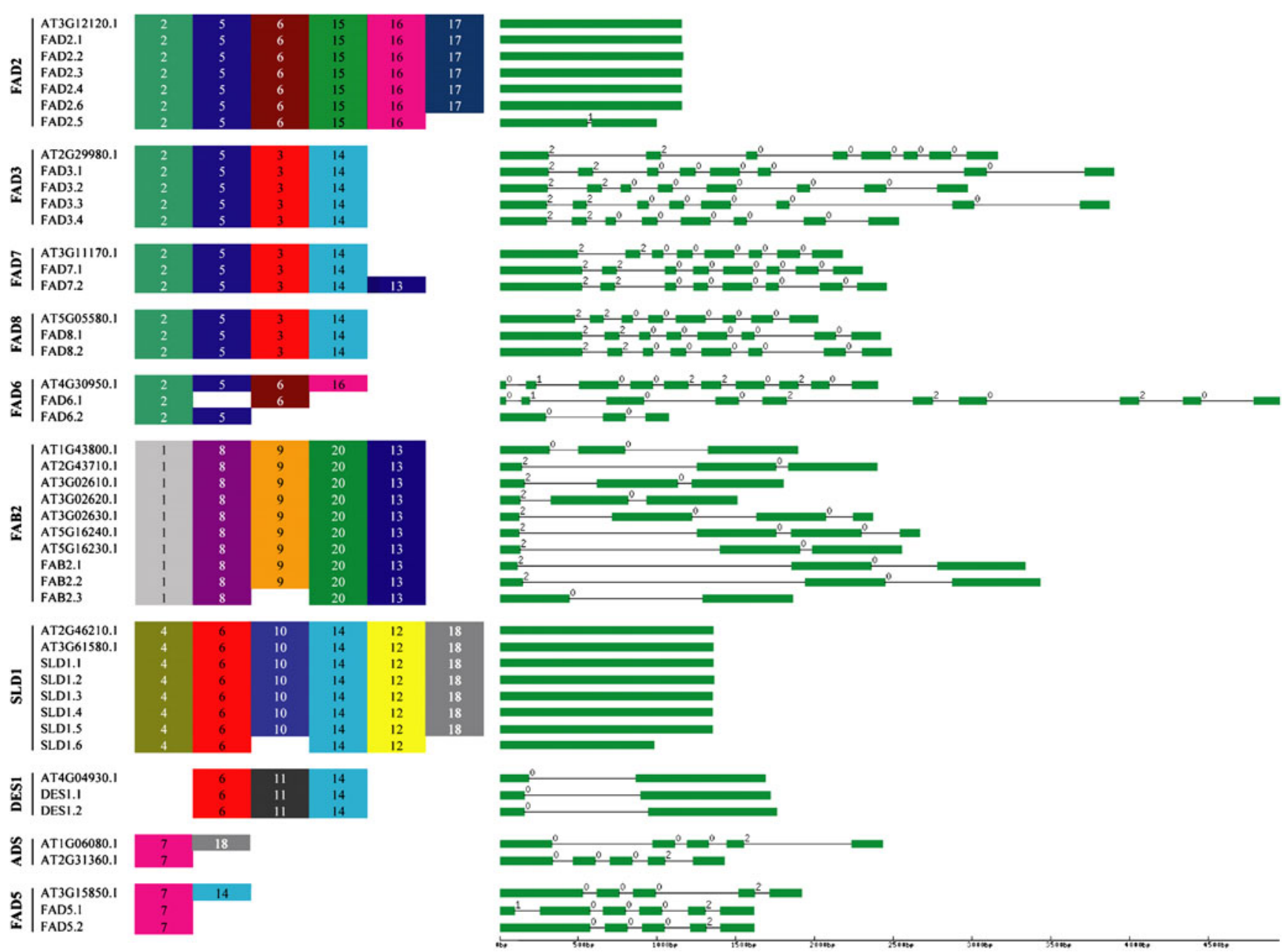

Fig. 2 The conserved motifs and exon/intron structures of the soybean and Arabidopsis fatty acid desaturase genes. Schematic representation of motifs identified in soybean fatty acid desaturase proteins using MEME motif search tool. Each motif was represented by a number in colored box. Length of box did not correspond to

The soluble desaturases are analyzed separately from membrane-bound desaturases because soluble desaturases are restricted to higher plants and show no evolutionary relationship with the more widely distributed membrane desaturases (Shanklin and Cahoon 1998). The desaturase

Table 4 SSR loci in fatty acid desaturase genes

\begin{tabular}{llcl}
\hline Locus tag & SSR type & SSR length & SSR location \\
\hline Glyma01g05470.1 & (AG)13 & 26 & 5' UTR \\
Glyma19g32940.1 & (TC)9 & 18 & Intron \\
Glyma02g15600.1 & (TAT)7 & 21 & Intron \\
Glyma03g30050.1 & (AT)13 & 26 & Extron \\
Glyma07g32850.1 & (TTA)7 & 21 & 5' UTR \\
Glyma07g32850.1 & (CT)17 & 34 & 5' UTR \\
Glyma08g22730.1 & (AACAC)5 & 25 & $5^{\prime}$ UTR \\
Glyma02g39230.1 & (AGC)5 & 15 & $5^{\prime}$ UTR \\
\hline
\end{tabular}

length of motif. Boxes represented the exons and lines represented introns. The sizes of exons and introns could be estimated using the scale at bottom. The numbers above the boxes and lines indicated the splicing phases of the fatty acid desaturase sequences, 0 referred to phase 0,1 to phase 1 , and 2 to phase 2

genes detected in soybean belonged to four subfamilies so far identified in other angiosperms: stearoyl-ACP desaturase subfamilies, $\Delta 7 / \Delta 9$ desaturase subfamilies, $\Delta 12 / \omega 3$ desaturase subfamilies, and "front-end" desaturase subfamilies ( $\Delta 4, \Delta 6$, and $\Delta 8$ desaturases).

The plant stearoyl-ACP desaturase is the only soluble desaturase identified to date. All other desaturases identified in plants, algae, animals, and fungi are integral membrane proteins (Singh et al. 2002; Luo et al. 2009). There were seven prospective stearoyl-ACP desaturases in Arabidopsis while soybean had five homologous genes that consisted of three full-length and two partial ones. The phylogenetic tree (Fig. 3) showed that the prospective stearoyl-ACP desaturases from soybean and Arabidopsis were grouped with those from higher plants and distinct from those of green algae. It may suggest that stearoyl-ACP desaturases in green algae and higher plants arose by independent gene duplication events. GmFAB2.2 


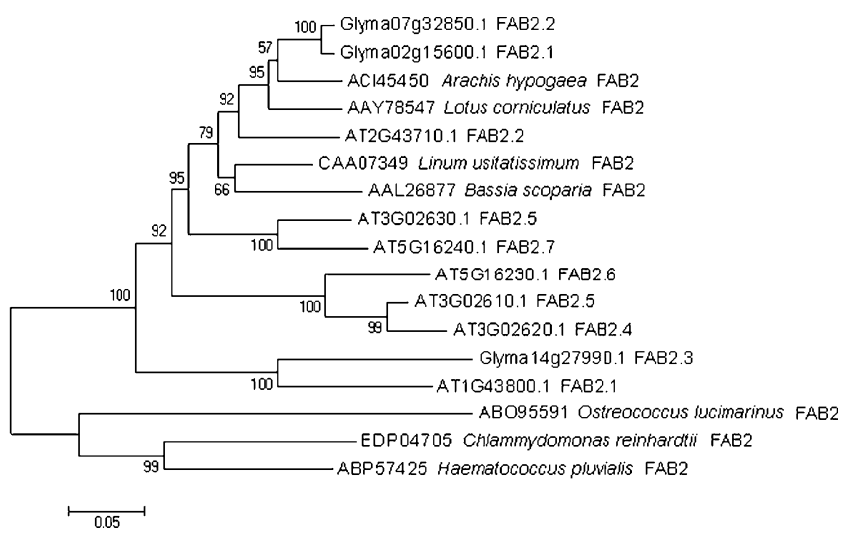

Fig. 3 Neighbor-joining tree based on the deduced amino acid sequences of stearoyl-acyl carrier protein desaturase homologs. Sequences from soybean and Arabidopsis genomes were shown by their locus tags and gene symbols. Other sequences were shown by their accession numbers, strain names, and gene symbols. Bootstrap values from neighbor-joining analyses were listed to the left of each node, with values more than 50 are shown

(GmFAB2A, Glyma07g32850) and GmFAB2.1 (GmFAB2B, Glyma02g15600) fell into a group and were constitutively expressed in both vegetative tissues and throughout seed development, whereas GmFAB2.3 (GmFAB2C, Glyma14g27990), clustered with FAB2.1 from Arabidopsis, were expressed only in developing seeds.

As shown in the phylogenetic tree, all of the membranebound desaturases fell into three distinct subfamilies: the $\Delta 7 / \Delta 9$ desaturase subfamily, $\Delta 12 / \omega 3$ desaturase subfamily, and "front-end" desaturase subfamily (Fig. 4). Based on functional criteria and the position of the clade integrated by $\Delta 9$ desaturases, $\Delta 9$ desaturase was assumed to be the ancestor of the remaining desaturases (Alonso et al. 2003). The $\Delta 7 / \Delta 9$ desaturase subfamily was clustered into two clades. In clade I, two $\triangle 7$-homologous genes from soybean (GmFAD5.1 and GmFAD5.2) as well as three genes (one $\Delta 7$ and two $\Delta 9$ ) from Arabidopsis grouped with $\Delta 7$ homologs from green algae, while the genes of cyanobacteria were placed in a basal position. Therefore, the $\Delta 9$ desaturase might arise by independent gene duplication events in plant and green algae branches, and the cyanobacterial $\Delta 9$ desaturase was identified as the origin of plant/green algae $\Delta 7 / \Delta 9$ desaturase. In clade II, one $\Delta 9$ homologous gene from Cyanidioschyzon merolae was grouped with those from fungi, apart from the subgroup comprised of genes from diatoms and animals.

In cluster I of the $\Delta 12 / \omega 3$ desaturase subfamily, two putative chloroplastic $\Delta 12$ desaturases from soybean (GmFAD6.1 and GmFAD6.2) and one from Arabidopsis formed a group with the desaturase genes from higher plants and green algae. Moreover, the $\Delta 12$ desaturases from cyanobacteria were situated at the basal position. In cluster II, six microsomal $\Delta 12$ desaturase genes from soybean as well as one gene from Arabidopsis clustered

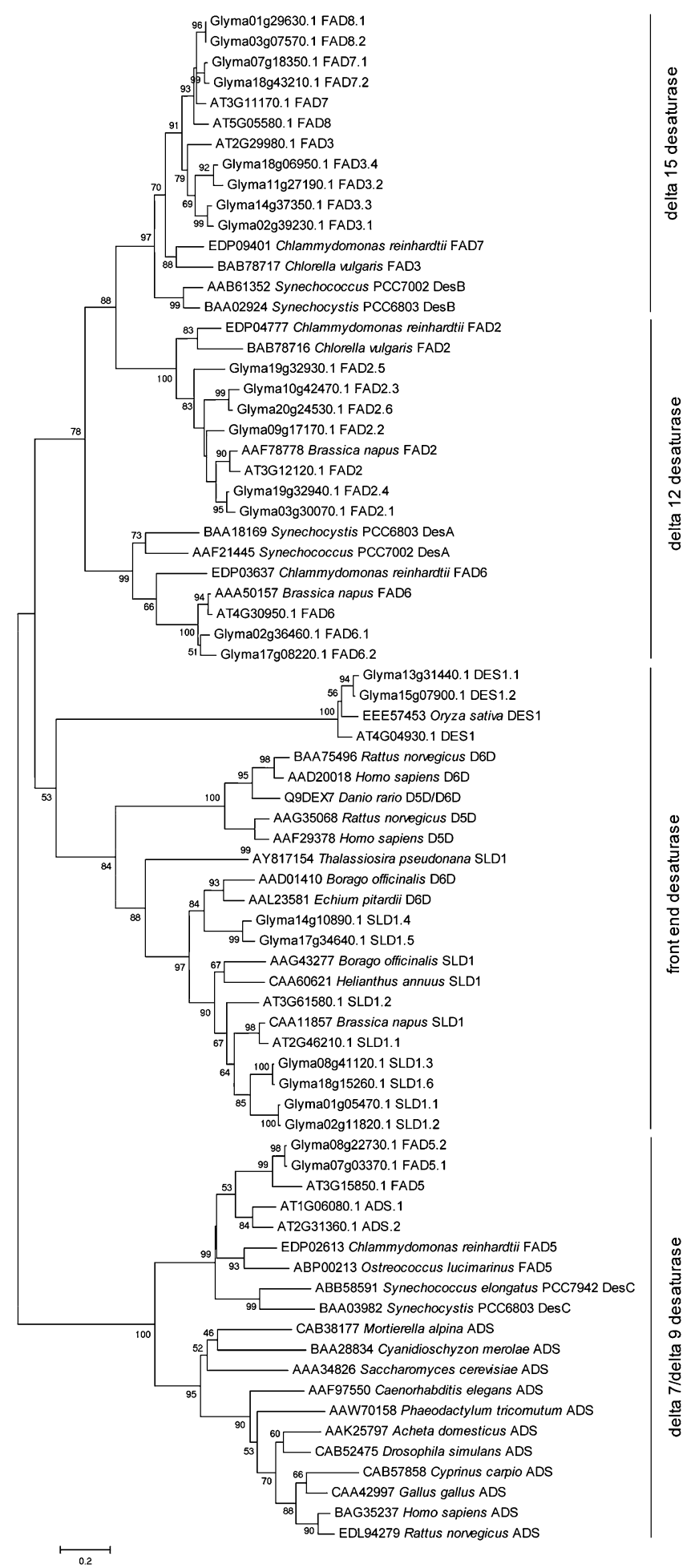

Fig. 4 Neighbor-joining tree of membrane desaturases. Sequences from soybean and Arabidopsis genomes were shown by their locus tags and gene symbols. Other sequences were shown by their accession numbers, strain names, and gene symbols. Bootstrap values from neighbor-joining analyses were listed to the left of each node, with values more than 50 are shown 
with genes from higher plants and green algae. Among FAD2-like genes that have been reported in soybean, two seed-specific genes GmFAD2.3 (GmFAD2-1A, Glyma10g42470) and GmFAD2.6 (GmFAD2-1B, Glyma20g24530) clustered together; two housekeeping genes GmFAD2.1 (GmFAD2-2C, Glyma03g30070) and GmFAD2.4 (GmFAD2-2B, Glyma19g32940) were grouped with FAD2 gene from Arabidopsis and set apart from GmFAD2.5 gene (GmFAD2-2A, Glyma19g32930). The GmFAD2.5 gene was not amplified from soybean by Schlueter et al. (2007), suggesting that GmFAD2.5 was either not expressed in tissue surveyed in this experiment or that this gene copy was no longer functional.

In cluster $\amalg$, the $\omega 3$ desaturases from cyanobacteria were placed in a basal position. Eight putative $\omega 3$ desaturases from soybean (four FAD3, two FAD8, and two FAD7) and three from Arabidopsis were grouped with their respective microsomal or chloroplastic $\omega 3$ desaturases from higher plants and set apart from enzymes from green algae. It was speculated that $\omega 3$ desaturases might arise by independent gene duplication events from a $\Delta 12$ desaturase ancestor, and the cyanobacterial $\Delta 12$ desaturase was identified as the origin of the plant $\omega 3$ desaturases, including both chloroplast and ER isozymes. Four alleles of GmFAD3 were separated into two groups: GmFAD3.1 (GmFAD3B, Glyma02g39230) and GmFAD3.3 (GmFAD3A, Glyma14g37350), GmFAD3.2 and GmFAD3.4 (GmFAD3C, Glyma18g06950). Although GmFAD3.1 and GmFAD3.3 showed high sequence similarity, GmFAD3.3 was the only gene that predominantly expressed in developing seeds (Bilyeu et al. 2003).

The "front-end" desaturases $(\Delta 4, \Delta 6$, and $\Delta 8$ desaturases) formed a separate clade (Fig. 4), and their phylogeny was complicated. The sphingolipid $\Delta 4$ desaturases from higher plants integrated into one group. In the other group, one subgroup was constituted by $\Delta 6$ desaturases from higher plants and $\Delta 8$-sphingolipid desaturases from higher plants and algae. Four putative $\Delta 8$ sphingolipid desaturases from soybean (GmSLD1.1, GmSLD1.2, GmSLD1.3 and GmSLD1.6), and two homologous genes from Arabidopsis integrated with those of higher plants, while two other soybean $\Delta 8$-sphingolipid desaturases (GmSLD1.4 and GmSLD1.5) clustered with $\Delta 6$ desaturases of higher plants. The other subgroup was integrated by $\Delta 5$ and $\Delta 6$ desaturases from animals.

\section{Coexpression Pattern Analysis}

Currently, there are 2,886 soybean and 4,013 Arabidopsis microarray chips available for query on the Genevestigator web site. The online analysis tools allow a large range of questions to be asked about gene expression during developmental stages, stress conditions, or by tissue/organ specificity (Grennan 2006). In this study, gene coexpression pattern analysis with 20 soybean and 16 Arabidopsis fatty acid desaturase genes was performed by the GENEVESTIGATOR program. Nine soybean desaturase genes and two Arabidopsis genes did not have their corresponding probe sets in the dataset (Table 5). The relatively low coverage of soybean desaturases in the datasets analyzed maybe partially due to the fact that these arrays were mainly derived from ESTs rather than offered whole genome coverage. From microarray based expression analysis, it was evident that 20 soybean and 16 Arabidopsis desaturase genes showed specific temporal and spatial expression patterns across different tissues and developmental stages (red and blue boxes, Fig. 5).

Figure 5a clustered organ-localized expression of the desaturase genes of Arabidopsis. The expression levels of AtSLD1.1, AtSLD1.2, and AtDES1 were very high in pollen and stamens relative to the expression of these genes in other organs suggesting a potentially specialized function in pollen development and/or function. AtFAD2, AtFAD3, and $A t F A B 2.5$ were extensively expressed among organs tested. AtFAD3 was expressed dominantly in callus, imbedded seed and endodermis cortex of root; AtFAD2 had relatively high expressions in pollen and stem, whereas AtFAB2.5 expressed strongly in stem and seed suspensor. AtFAD5, AtFAD6, AtFAD7, and AtFAB2.2 had similar expression patterns, showing higher transcript abundance in seedling cotyledons, stem, adult leaf, and leaf primordia. AtFAB2.1 preferentially expressed in callus, endodermis cortex, and lateral root cap of roots, whereas AtADS.2 exhibited specifically high transcript accumulation in chalazal endosperm and testa of seed. Figure $5 \mathrm{c}$ reported expression of the Arabidopsis desaturase genes over nine stages of plant development. AtFAB2.3, AtFAB2.6, AtSLD1.1, and AtDES1 RNAs were found in low abundance over all stages, whereas AtFAD5 had relatively high expression. AtADS.1 and AtFAB2.7 transcripts were relatively rare; AtADS.1 expression was limited to primarily germinated seeds (Fig. 5c "stage 1"), whereas AtFAB2.7 was largely restricted to mature siliques (Fig. 5c "stage 9"). AtFAD6 and AtFAD7 shared the characteristic of having maximal expression very early and expressing at their lowest levels at the latest developmental stage. AtADS.2, AtFAB2.2, AtFAB2.5, AtFAD2, AtFAD3, and AtSLD1.2 had similar expression behavior over the developmental stages with higher expression in developed flower (Fig. 5c "stage 7"); AtFAB2.2, AtFAD2, and AtFAD3 were expressed at their highest levels at the earliest developmental stage; in addition, AtADS.2, AtFAB2.2, AtFAD2.5, and AtFAB3 had relatively high expression at the latest developmental stage.

Figure $5 \mathrm{~b}$ showed the organ-specific coexpression between different desaturase genes of soybean. Six gene pairs (GmSLD1.1/GmSLD1.2, GmFAD8.1/GmFAD8.2, GmFAD6.1/GmFAD6.2, GmSLD1.3/GmSLD1.6, GmDES1.1/GmDES1.2, GmSLD1.4/GmSLD1.5) had the 
Table 5 Selected fatty acid desaturase genes used for coexpression analysis in GENEVESTIGATOR

\begin{tabular}{|c|c|c|c|c|}
\hline Organism & Gene symbol & Locus tag & UniGene number & Microarray probe \\
\hline \multirow[t]{29}{*}{ Glycine max } & SLD1.1 & Glyma01g05470.1 & Gma.20380 & GmaAffx.93332.1.S 1 at \\
\hline & FAD8.1 & Glyma01g29630.1 & Gma.11043 & Gma.11043.1.S 1 at \\
\hline & SLD1.2 & Glyma02g11820.1 & Gma.20380 & GmaAffx.93332.1.S 1_at \\
\hline & FAB2.1 & Glyma02g15600.1 & Gma.32339 & Gma.6256.1.S1_S_at \\
\hline & FAD6.1 & Glyma02g36460.1 & Gma.49 & Gma.49.1.S ${ }_{1 \_}$at \\
\hline & FAD3.1 & Glyma02g39230.1 & Gma.50724 & / \\
\hline & FAD2.1 & Glyma03g30070.1 & Gma.25376 & GmaAffx.93278.1.S1_S_at \\
\hline & FAD8.2 & Glyma03g07570.1 & Gma.11043 & Gma.11043.1.S 1_at \\
\hline & FAB2.2 & Glyma07g32850.1 & Gma.6256 & GmaAffx.43163.1.S S_at \\
\hline & FAD5.1 & Glyma07g03370.1 & Gma.22389 & / \\
\hline & FAD7.1 & Glyma07g18350.1 & Gma.9403 & / \\
\hline & SLD1.3 & Glyma08g41120.1 & Gma.7596 & Gma.7596.1.S. \\
\hline & FAD5.2 & Glyma08g22730.1 & Gma.22389 & / \\
\hline & FAD2.2 & Glyma09g17170.1 & / & / \\
\hline & FAD2.3 & Glyma10g42470.1 & Gma.18367 & Gma.1839.1.S $\mathrm{S}_{1}$ at \\
\hline & FAD3.2 & Glyma11g27190.1 & Gma.8463 & / \\
\hline & DES1.1 & Glyma13g31440.1 & Gma.5077 & Gma.5077.1.A 1 _at \\
\hline & SLD1.4 & Glyma14g10890.1 & Gma.19021 & GmaAffx.87656.1.S S_at $_{\text {a }}$ \\
\hline & FAB2.3 & Glyma14g27990.1 & Gma.36064 & / \\
\hline & FAD3.3 & Glyma14g37350.1 & Gma.30186 & Gma.10564.1.1.S $\mathrm{S}_{1}$ at \\
\hline & DES1.2 & Glyma15g07900.1 & Gma.5077 & 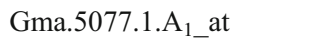 \\
\hline & SLD1.5 & Glyma17g34640.1 & Gma.19021 & GmaAffx.87656.1.S 1_at \\
\hline & FAD6.2 & Glyma17g08220.1 & Gma.49 & Gma.49.1.S 1_at $_{-}$ \\
\hline & SLD1.6 & Glyma18g15260.1 & Gma.7596 & Gma.7596.1.S. \\
\hline & FAD7.2 & Glyma18g43210.1 & Gma.9403 & / \\
\hline & FAD3.4 & Glyma18g06950.1 & Gma.26862 & Gma.8463.1.S $\mathrm{S}_{1 \_ \text {at }}$ \\
\hline & FAD2.4 & Glyma19g32940.1 & Gma.5046 & GmaAffix.90006.1.A_ at \\
\hline & FAD2.5 & Glyma19g32930.1 & / & / \\
\hline & FAD2.6 & Glyma20g24530.1 & Gma.1839 & Gma.1839.2.S 1 _a_at \\
\hline \multirow[t]{18}{*}{ Arabidopsis thaliana } & FAB2.1 & AT1G43800.1 & At.43113 & 260869_at \\
\hline & FAB2.2 & AT2G43710.1 & At. 22018 & 260570_at \\
\hline & FAB2.3 & AT3G02610.1 & At.41109 & 258473_s_at \\
\hline & FAB2.4 & AT3G02620.1 & At.41106 & / \\
\hline & FAB2.5 & AT3G02630.1 & At. 18818 & 258485_at \\
\hline & FAB2.6 & AT5G16230.1 & At. 31698 & 246498_at \\
\hline & FAB2.7 & AT5G16240.1 & At.26326 & 246502_at \\
\hline & ADS.1 & AT1G06080.1 & At. 342 & 260957_at \\
\hline & ADS.2 & AT2G31360.1 & At. 14455 & 263249_at \\
\hline & FAD2 & AT3G12120.1 & At. 23898 & 256277_at \\
\hline & FAD6 & AT4G30950.1 & At.1931 & 253547_at \\
\hline & FAD7 & AT3G11170.1 & At. 272 & 256417_s_at \\
\hline & FAD8 & AT5G05580.1 & At. 273 & / \\
\hline & FAD3 & AT2G29980.1 & At. 271 & 266865_at \\
\hline & FAD5 & AT3G15850.1 & At. 5631 & 258250_at \\
\hline & SLD1.1 & AT2G46210.1 & At.36539 & 266592_at \\
\hline & SLD1.2 & AT3G61580.1 & At. 120 & 251323_at \\
\hline & DES1 & AT4G04930.1 & At.4051 & 255276_at \\
\hline
\end{tabular}




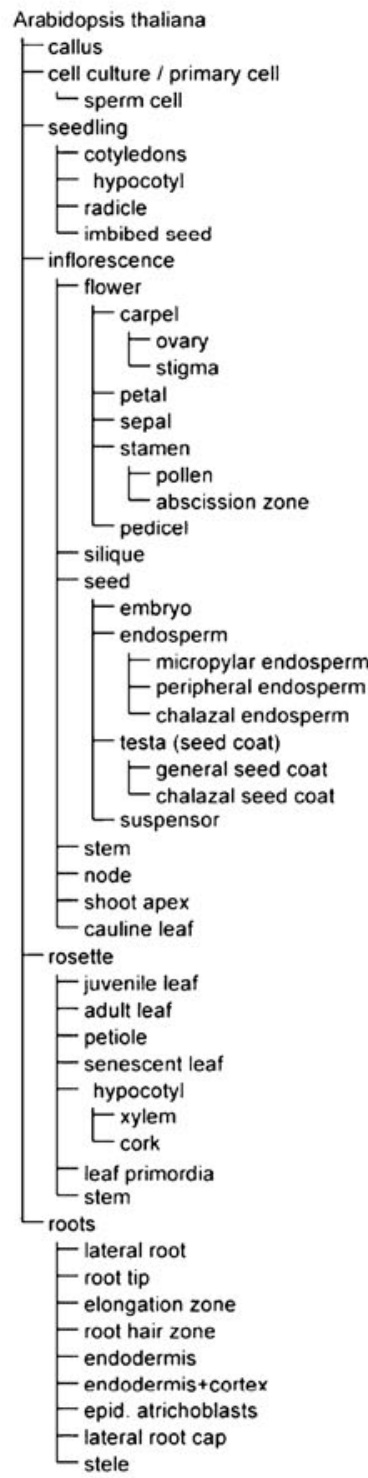

A

nกตก nก N
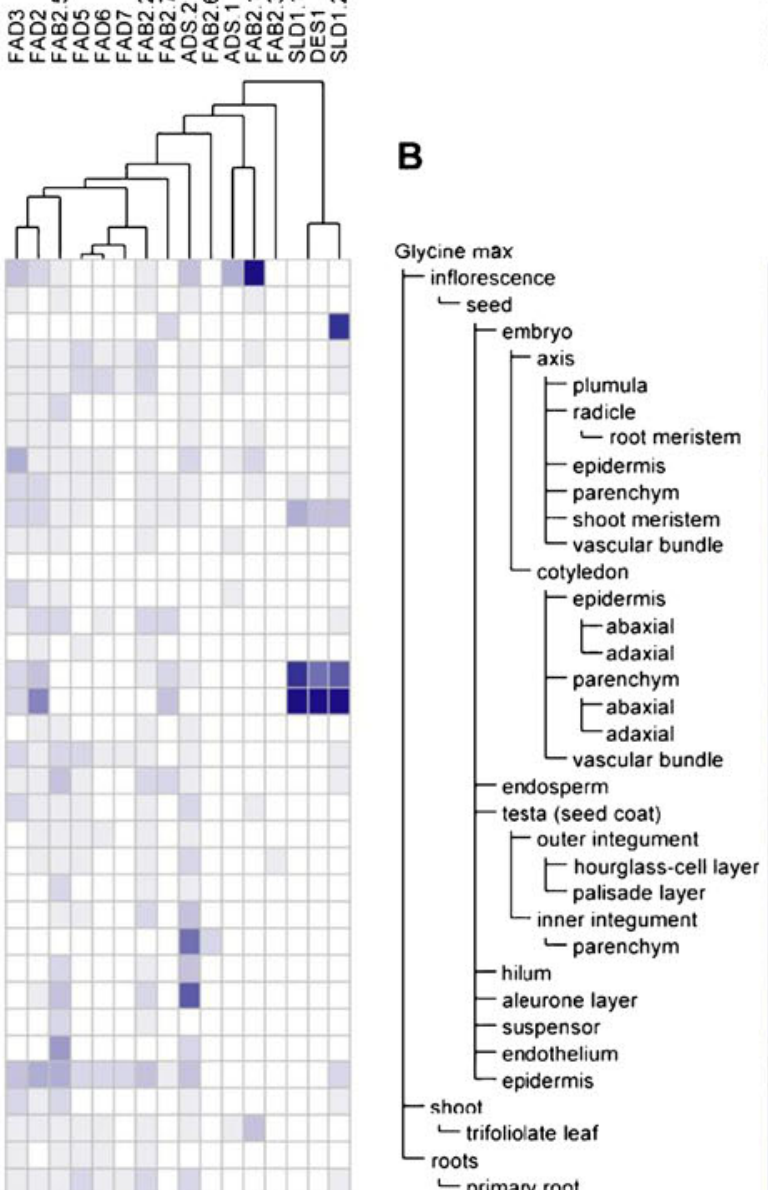

D

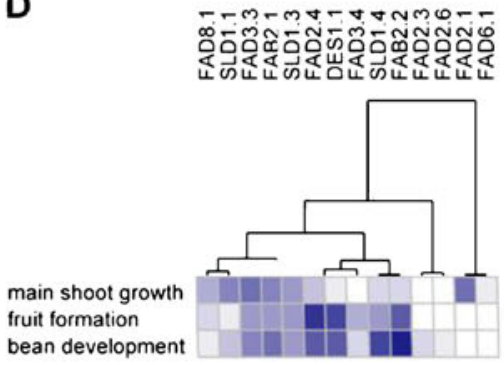

C

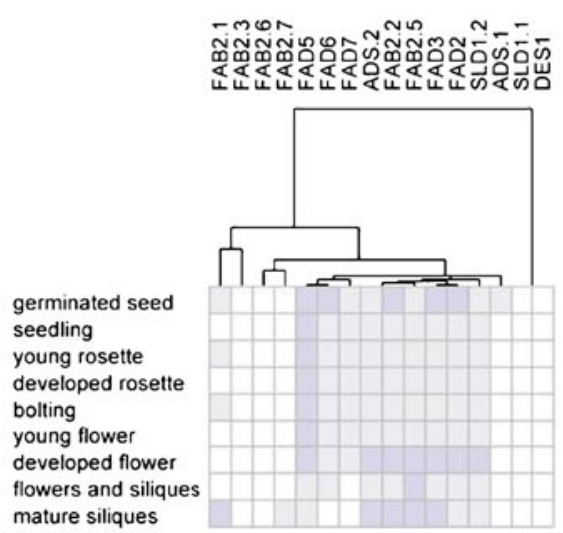


Fig. 5 xThe coexpression analysis of selected fatty acid desaturase genes. The Arabidopsis and soybean desaturase genes were analyzed for organ-specific and developmental coexpression patterns by GENEVESTIGATOR program. The hierarchical clustering was calculated by Pearson correlation. a, c Hierarchical clustering of organ-specific and developmental expression profiles of 16 desaturase genes of Arabidopsis. b, d Hierarchical clustering of organ-specific and developmental expression profiles of 20 desaturase genes of soybean. In soybean six gene pairs (SLD1.1/SLD1.2, FAD8.1/FAD8.2, FAD6.1/FAD6.2, SLD1.3/SLD1.6, DES1.1/DES1.2, SLD1.4/ SLD1.5) shared the same expression patterns with respect to the organs and developmental stages examined, respectively

same probe sets and shared the same expression patterns, respectively. GmFAD2.3, GmFAD2.6, and GmFAD3.4 had dedicated expression in seeds; the expression patterns of GmFAD2.3 and GmFAD2.6 were similar, expressing very strongly in cotyledon parenchyma, whereas GmFAD3.4 showed the highest transcript abundance in inner integument of testa among tissues tested. GmFAD2.1, GmFAD8.1/ GmFAD8.2, and GmFAD6.1/GmFAD6.2 had different tissuespecific expression patterns, mainly expressed in trifoliolate leaf. The remaining eight desaturase genes were largely restricted in their expression to seeds, mainly expressed higher in embryo. Figure 5d summarized developmental expression profiles of desaturase genes of soybean over three stages. GmFAD3.3, GmFAB2.1, and GmSLD1.3/GmSLD1.6 mRNAs were found in high abundance over all stages. GmFAD2.3 and GmFAD2.6 expressions were limited to bean development stage (Fig. 5d "stage 3"); in contrast, GmFAD2.1 and GmFAD6.1/GmFAD6.2 were largely restricted to main shoot growth stage (Fig. 5d "stage 1"). The expression levels of GmFAD3.4, GmFAB2.2, GmSLD1.4/GmSLD1.5, and GmDES1.1/GmDES1.2 remained relatively low at the initial stage but showed dramatic increases in abundance during later stages, whereas GmFAD8.1/GmFAD8.2 and GmSLD1.1/ GmSLD1.2 showed the highest transcript abundance in the main shoot growth stage and decreased thereafter.

It was noteworthy that several desaturase orthologs between Arabidopsis and soybean showed strikingly consistent expression patterns, which lent further supporting to the existence of functional conservation between these two species. For example, the expression profiles of homologous pair GmFAD6.1 and GmFAD6.2 highly resembled the expression pattern of their closest ortholog AtFAD6 in Arabidopsis, with preferential expressions in photosynthetic tissues. However, there still existed some differences. Both GmFAD7 paralogous genes (GmFAD7-1 and GmFAD7-2) have been functionally characterized and expressed in all soybean tissues analyzed (Andreu et al. 2010), whereas in Arabidopsis no AtFAD7 mRNA was detected in roots (Yadav et al. 1993; Nishiuchi et al. 1997).

Many genes encoding both microsomal and plastidial fatty acid desaturases have been isolated and studied from soybean. These genes showed very distinct tissue-specific expression patterns, which were in good agreement with the above microarray profiles. For example, in accordance with previous findings, GmFAD2.3 (GmFAD2-1A, Glyma10g42470) and GmFAD2.6 (GmFAD2-1B, Glyma20g24530) were specifically expressed in developing seeds (Heppard et al. 1996). Consistent with the microarray profiles, GmFAD2.4 (GmFAD2-2B, Glyma19g32940) was expressed in all tissues surveyed; GmFAD2.1 (GmFAD2-2C, Glyma03g30070) had constitutively weak expression levels in seed grown in warm conditions, and it showed upward of an eightfold increase in expression in developing seeds grown in cooler conditions (Schlueter et al. 2007).

\section{Conclusion}

Contribution of fatty acid desaturase family for the functional properties of both animal fats and vegetable oils is well documented (Kinney et al. 2002). However, only a limited number of fatty acid desaturase genes have been functionally validated in soybean by now. In the present study, a comprehensive analysis including phylogeny, chromosomal locations, gene structures, conserved motifs, and expression profiling of desaturase family in soybean was performed. We identified a sum of 29 full-length desaturase genes in soybean genome. Twelve additional partial desaturase sequences could represent additional genes. The desaturase genes in Arabidopsis were phylogenetically clustered into ten distinct subfamilies. Representatives of all except $A D S$ subfamily were found in soybean. The exon/intron structures and motif compositions of desaturases were highly conserved in each subfamily, indicative of their functional conservation. The comprehensive annotation undertaken in this investigation improved our understanding of the involvement of these genes in fatty acid synthesis in soybean and provided the source for selection of candidate genes for functional validation studies.

Acknowledgments This work was supported by a grant from Modern Agro-industry Technology Research System (nycytx-19), National High-Tech Research and Development Plan of China (No.2006AA10A114 and 2007AA10Z189), National Project of Scientific and Technical Supporting Program (2008BAD97B04), the National Natural Science Foundation of China (31000728), and the Natural Science Fund of Shangdong Province (ZR2009DQ004).

\section{References}

Alonso DL, Maroto FG, Ruiz JR, Garrido JA, Vilches MA (2003) Evolution of the membrane-bound fatty acid desaturases. Biochem Syst Ecol 31:1111-1124

Andreu V, Lagunas B, Collados R, Picorel R, Alfonso M (2010) The GmFAD7 gene family from soybean: identification of novel genes and tissue-specific conformations of the FAD7 enzyme 
involved in desaturase activity. J Exp Bot 61:3371-3384. doi: $10.1093 / \mathrm{jxb} / \mathrm{erq} 158$

Bailey TL, Elkan C (1995) The value of prior knowledge in discovering motifs with MEME. Proc Int Conf Intell Syst Mol Biol 3:21-29

Bilyeu KD, Palavalli L, Sleper DA, Beuselinck PR (2003) Three microsomal omega-3 fatty-acid desaturase genes contribute to soybean linolenic acid levels. Crop Sci 43:1833-1838

Bowman JL, Floyd SK, Sakakibara K (2007) Green genes - comparative genomics of the green branch of life. Cell 129:229-234

Byfield GE, Xue H, Upchurch RG (2006) Two genes from soybean encoding soluble $\Delta 9$ stearoyl-ACP desaturases. Crop Sci 46:840-846

Chen Z, Wang M, Barkley NA, Pittman RN (2010) A simple allelespecific PCR assay for detecting FAD2 alleles in both A and B genomes of the cultivated peanut for high-oleate trait selection. Plant Mol Biol Report 28:542-548. doi:10.1007/s11105-0100181-5

Chi X, Yang Q, Zhao F, Qin S, Yang Y, Shen J, Lin H (2008a) Comparative analysis of fatty acid desaturases in cyanobacterial genomes. Comp Funct Genomics 2008:284508. doi:10.1155/ 2008/284508

Chi X, Zhang X, Guan X, Ding L, Li Y, Wang M, Qin S (2008b) Fatty acid biosynthesis in eukaryotic photosynthetic microalgae: identification of a microsomal delta 12 desaturase in Chlamydomonas reinhardtii. J Microbiol 46:189-201

Dornbos DL Jr, Mullen RE (1992) Soybean seed protein and oil contents and fatty acid composition adjustments by drought and temperature. J Am Oil Chem Soc 69:228-231

Flores T, Karpova O, Su ZJ, Zeng PY, Bilyeu K, Sleper DA, Nguyen HT, Zhang ZYJ (2008) Silencing of GmFAD3 gene by siRNA leads to low $\alpha$-linolenic acids (18:3) of fad3-mutant phenotype in soybean [Glycine $\max$ (Merr.)]. Transgenic Res 17:839-850

Grennan AK (2006) Genevestigator. facilitating web-based geneexpression analysis. Plant Physiol 141:1164-1166

Guo AY, Zhu QH, Chen X, Luo JC (2007) GSDS: a gene structure display server. Yi Chuan 29:1023-1026

Heppard EP, Kinney AJ, Stecca KL, Miao GH (1996) Developmental and growth temperature regulation of two different microsomal omega-6 desaturase genes in soybeans. Plant Physiol 110:311319

Kinney AJ, Cahoon EB, Hitz WD (2002) Manipulating desaturase activities in transgenic crop plants. Biochem Soc Trans 30:1099-1103

Li LY, Wang XL, Gai JY, Yu DY (2007) Molecular cloning and characterization of a novel microsomal oleate desaturase gene from soybean. J Plant Physiol 164:1516-1526

Li M, Wang X, Su L, Bi Y, Wan S (2010) Characterization of five putative acyl carrier protein (ACP) isoforms from developing seeds of Arachis hypogaea L. Plant Mol Biol Report 28:365372. doi:10.1007/s11105-009-0160-x

Lu Y, Chi X, Li Z, Yang Q, Li F, Liu S, Gan Q, Qin S (2010) Isolation and characterization of a stress-dependent plastidial $\Delta^{12}$ fatty acid desaturase from the Antarctic microalga Chlorella vulgaris NJ-7. Lipids 45:179-187

Lu Y, Chi X, Yang Q, Li Z, Liu S, Gan Q, Qin S (2009) Molecular cloning and stress-dependent expression of a gene encoding $\Delta^{12}$ fatty acid desaturase in the Antarctic microalga Chlorella vulgaris NJ-7. Extremophiles 13:875-884

Luo T, Deng WY, Zeng J, Zhang FL (2009) Cloning and characterization of a stearoyl-acyl carrier protein desaturase gene from Cinnamomum longepaniculatum. Plant Mol Biol Report 27:13-19. doi:10.1007/s11105-008-0046-3

Nishiuchi T, Hamada T, Kodama H, Iba K (1997) Wounding changes the spatial expression pattern of the Arabidopsis plastid $\omega 3$ fattyacid desaturase gene (FAD7) through different signal transduction pathways. Plant Cell 9:1701-1712
Ohlrogge J, Browse J (1995) Lipid biosynthesis. Plant Cell 7:957970

Pattee HE, Isleib TG, Moore KM, Gorbet DW, Giesbrecht FG (2002) Effect of high-oleic trait and paste storage variables on sensory attribute stability of roasted peanuts. J Agr Food Chem 50:73667370

Salamov AA, Solovyev VV (2000) Ab initio gene finding in Drosophila genomic DNA. Genome Res 10:516-522

Saski C, Lee SB, Daniell H, Wood TC, Tomkins J, Kim HG, Jansen RK (2005) Complete chloroplast genome sequence of Glycine max and comparative analyses with other legume genomes. Plant Mol Biol 59:309-322

Sato N, Moriyama T (2007) Genomic and biochemical analysis of lipid biosynthesis in the unicellular rhodophyte Cyanidioschyzon merolae: lack of a plastidic desaturation pathway results in the coupled pathway of galactolipid synthesis. Eukaryot Cell 6:1006-1017

Schlueter JA, Vasylenko-Sanders IF, Deshpande S, Yi J, Siegfried M, Roe BA, Schlueter SD, Scheffler BE, Shoemaker RC (2007) The FAD2 gene family of soybean: insights into the structural and functional divergence of a paleopolyploid genome. Crop Sci 47 (S1):S14-S26

Schmutz J, Cannon SB, Schlueter J et al (2010) Genome sequence of the palaeopolyploid soybean. Nature 463:178-183

Senthilvel S, Jayashree B, Mahalakshmi V, Kumar PS, Nakka S, Nepolean T, Hash C (2008) Development and mapping of simple sequence repeat markers for pearl millet from data mining of expressed sequence tags. BMC Plant Biol 27:119. doi:10.1186/ 1471-2229-8-119

Shanklin J, Cahoon EB (1998) Desaturation and related modifications of fatty acids. Annu Rev Plant Physiol Plant Mol Biol 49:611641

Shilman F, Brand Y, Brand A, Hedvat I, Hovav R (2010) Identification and molecular characterization of homeologous $\Delta 9$-stearoyl acyl carrier protein desaturase 3 genes from the allotetraploid peanut (Arachis hypogaea). Plant Mol Biol Report. doi:10.1007/s11105-010-0226-9

Singh SC, Sinha RP, Häder DP (2002) Role of lipids and fatty acids in stress tolerance in cyanobacteria. Acta Protozool 41:297-308

Sturn A, Quackenbush J, Trajanoski Z (2002) Genesis: cluster analysis of microarray data. Bioinformatics 18:207-208

Tamura K, Dudley J, Nei M, Kumar S (2007) MEGA4: molecular evolutionary genetics analysis (MEGA) software version 4.0. Mol Biol Evol 24:596-1599

Tang GQ, Novitzky WP, Griffin HC, Hube SC, Dewey RE (2005) Oleate desaturase enzymes of soybean: evidence of regulation through differential stability and phosphorylation. Plant J 44:433-446

Tao F, Zhu SW, Fan J, Cheng BJ (2006) Cloning and sequence analysis of maize FAD2 gene. J Plant Physiol Mol Biol 32:649-656

Thiel T, Michalek W, Varshney RK, Graner A (2003) Exploiting EST databases for the development and characterization of genederived SSR-markers in barley (Hordeum vulgare L.). Theor Appl Genet 106(3):411-422

Thompson JD, Higgins DG, Gibson TJ (1994) CLUSTALW: improving the sensitivity of progressive multiple sequence alignment through sequence weighting, position-specific gap penalties and weight matrix choice. Nucleic Acids Res 22:4673-4680

Topfer R, Martini N, Schell J (1995) Modification of plant lipid synthesis. Science 268:681-688

Varshney RK, Graner A, Sorrells ME (2005) Genic microsatellite markers in plants: features and applications. Trends Biotechnol 23(1):48-55

Vijay D, Dadlani M, Kumar PA, Panguluri SK (2009) Molecular marker analysis of differentially aged seeds of soybean and 
safflower. Plant Mol Biol Report 27:282-291. doi:10.1007/ s11105-008-0085-9

William DH, Thomas JC, Russell Booth J, Jr Anthony JK, Kevin LS, Narendra SY (1994) Cloning of a higher-plant plastid $\omega-6$ fatty acid desaturase cDNA and its expression in a cyanobacterium. Plant Physiol 105:635-641

Yadav NS, Wierzbicki A, Aegerter M, Caster CS, Pérez-Grau L, Kinney AJ, Hitz WD, Booth JR Jr, Schweiger B, Stecca KL (1993) Cloning of higher plant $\omega 3$ fatty-acid desaturases. Plant Physiol 103:467-476

Yu J, Dake TM, Singh S, Benscher D, Li W, Gill B, Sorrells ME (2004) Development and mapping of EST-derived simple sequence repeat markers for hexaploid wheat. Genome 47:805-818
Zhang C, Meng Q, Zhang M, Huang F, Gai J, Yu D (2008a) Characterization of o-acetylserine(thiol)lyase-encoding genes reveals their distinct but cooperative expression in cysteine synthesis of soybean [Glycine max (L.) Merr.]. Plant Mol Biol Report 26:277-291. doi:10.1007/s11105-008-0047-2

Zhang P, Burton JW, Upchurch RG, Whittle E, Shanklin J, Dewey RE (2008b) Mutations in a $\Delta^{9}$-stearoyl-ACP-desaturase gene are associated with enhanced stearic acid levels in soybean seeds. Crop Sci 48:2305-2313

Zimmermann P, Hennig L, Gruissem W (2005) Gene-expression analysis and network discovery using Genevestigator. Trends Plant Sci 10:407-409 